\title{
Edema following Gamma Knife radiosurgery for parasagittal and parafalcine meningiomas
}

\author{
Jason P. Sheehan, MD, PhD, ${ }^{1,2}$ Cheng-Chia Lee, MD, ${ }^{1}$ Zhiyuan Xu, MD, ${ }^{1}$ Colin J. Przybylowski, BS, ${ }^{1}$ \\ Patrick D. Melmer, BA, ${ }^{1}$ and David Schlesinger, $\mathrm{PhD}^{1,2}$ \\ Departments of ${ }^{1}$ Neurological Surgery and ${ }^{2}$ Radiation Oncology, University of Virginia Health System, Charlottesville, Virginia
}

OBJECT Stereotactic radiosurgery (SRS) has been shown to offer a high probability of tumor control for Grade I meningiomas. However, SRS can sometimes incite edema or exacerbate preexisting edema around the targeted meningioma. The current study evaluates the incidence, timing, and degree of edema around parasagittal or parafalcine meningiomas following SRS.

METHODS A retrospective review was undertaken of a prospectively maintained database of patients treated with Gamma Knife radiosurgery at the University of Virginia Health System. All patients with WHO Grade I parafalcine or parasagittal meningiomas with at least 6 months of clinical follow-up were identified, resulting in 61 patients included in the study. The median radiographic follow-up was 28 months (range 6-158 months). Rates of new or worsening edema were quantitatively assessed using volumetric analysis; edema indices were computed as a function of time following radiosurgery. Statistical methods were used to identify favorable and unfavorable prognostic factors for new or worsening edema.

RESULTS Progression-free survival at 2 and 5 years was $98 \%$ and $90 \%$, respectively, according to Kaplan-Meier analysis. After SRS, new peritumoral edema occurred or preexisting edema worsened in $40 \%$ of treated meningiomas. The median time to onset of peak edema was 36 months post-SRS. Persistent and progressive edema was associated with 11 tumors, and resection was undertaken for these lesions. However, 20 patients showed initial edema progression followed by regression at a median of 18 months after radiosurgery (range 6-24 months). Initial tumor volume greater than $10 \mathrm{~cm}^{3}$, absence of prior resection, and higher margin dose were significantly $(p<0.05)$ associated with increased risk of new or progressive edema after SRS.

CONCLUSIONS Stereotactic radiosurgery offers a high rate of tumor control in patients with parasagittal or parafalcine meningiomas. However, it can lead to worsening peritumoral edema in a minority of patients. Following radiosurgery, transient edema occurs earlier than persistent and progressive edema. Longitudinal follow-up of meningioma patients after SRS is required to detect and appropriately treat transient as well as progressive edema.

http://thejns.org/doi/abs/10.3171/2014.12.JNS142159

KEY WORDS stereotactic radiosurgery; Gamma Knife; meningioma; edema

$\mathrm{S}$ TEREOTACTIC radiosurgery (SRS) is frequently used as an option to treat patients with parasagittal or parafalcine meningiomas. ${ }^{5}$ Radiosurgery is typically chosen to treat patients for meningiomas that are $3 \mathrm{~cm}$ or smaller in largest dimensions and for whom a resection would provide a high risk of morbidity. SRS may be used as an upfront treatment or as an adjuvant one to treat a recurrent or residual meningioma. Most studies show that radiosurgery affords long-term tumor control in greater than $85 \%$ of patients harboring a WHO Grade I parasagittal/parafalcine meningioma. ${ }^{5}$

Meningiomas involving the parasagittal or parafalcine region frequently abut or even invade venous structures. The proximity of the tumor to a sinus or draining vein can lead to venous congestion. Venous obstruction, pial supply, location, tumor volume, tumor grade and histological subtype, vascular growth factor receptor expression, and patient age have been associated with peritumoral edema

ABBREVIATIONS GKRS = Gamma Knife radiosurgery; SRS = stereotactic radiosurgery; VEGF = vascular endothelial growth factor SUBMITTED September 15, 2014. ACCEPTED December 17, 2014.

INCLUDE WHEN CITING Published online June 26, 2015; DOI: 10.3171/2014.12.JNS142159.

DISCLOSURE Dr. Schlesinger reports receiving support of non-study-related research from Elekta AB. 
in meningiomas., ${ }^{7,17,21}$ Peritumoral edema associated with parasagittal or parafalcine meningiomas is common, with some series showing edema in $48 \%$ of patients. ${ }^{17}$ At times, the edema may be symptomatic and necessitate medical therapy such as steroids to lessen or relieve symptoms. However, in other cases, the edema may be seen as T2weighted changes on MRI, but the patient may remain asymptomatic.

Following SRS, edema can worsen in a delayed fashion. Patients with symptoms may experience progression, and asymptomatic patients with edema may become symptomatic. Post-SRS edema has been noted in as many as $7 \%-38 \%$ of cases. ${ }^{2,8,10,16,20,22,26}$ In the current study, we evaluate the risk of postradiosurgical edema in a quantitative fashion and determine the factors associated with its occurrence in patients with parasagittal or parafalcine meningiomas.

\section{Methods}

\section{Patient Population}

We reviewed a prospectively collected database approved by the University of Virginia institutional review board and identified patients with parasagittal or parafalcine meningiomas treated with Gamma Knife radiosurgery (GKRS) between 1991 and 2013. Patients who had undergone prior resection of a WHO Grade I meningioma were included in the study, while patients with a WHO Grade II or III meningioma were excluded from the current study. Patients with clinical and imaging features characteristic of a benign meningioma involving the parasagittal or parafalcine region and no history of previous cancer were also included in the study. For inclusion, the patient's GKRS dose-planning MRI study and at least 1 post-GKRS MRI study with thin slices (5 $\mathrm{mm}$ or less) acquired through the volume of the head must have been available to permit volumetric analysis of the tumor and the peritumoral edema. Patients with only CT imaging were excluded from the study.

Table 1 summarizes the 61 patients included in the study. Forty-eight patients had 1 discrete meningioma, 10 patients had 2 discrete meningiomas, and 3 patients had 3 discrete meningiomas that were treated with GKRS. In terms of local tumor control and edema response, these tumors were analyzed separately. The mean age of the patients in the study was 59.7 years (range 18-81.6 years). The most common clinical presentations were headache in 22 cases (29\%), motor deficit in 19 cases (25\%), seizures in 19 cases (25\%), and visual disturbances in 13 cases (17\%).

\section{Tumor Characteristics}

Of the 77 meningiomas included for imaging analysis, 43 were parasagittal $(55.8 \%)$ and 34 were parafalcine $(44.2 \%)$. The median initial tumor volume was 5.6 $\mathrm{cm}^{3}$ (range $0.3-17.5 \mathrm{~cm}^{3}$ ). Treatment prior to radiosurgery treatment included resection for 62 of the tumors $(80.5 \%)$. Tumor attributes are detailed in Table 1.

\section{Gamma Knife Radiosurgery Technique}

The GKRS technique used at our institution has been previously described. ${ }^{5}$ For the current series, radiosurgical
TABLE 1. Characteristics in 61 patients with 77 intracranial meningiomas treated with SRS

\begin{tabular}{|c|c|}
\hline Attribute & Value* \\
\hline \multicolumn{2}{|l|}{ Age in yrs } \\
\hline Median & 59.7 \\
\hline Range & $18.0-81.6$ \\
\hline Sex (F/M) & $37: 24$ \\
\hline \multicolumn{2}{|l|}{ No. of tumors } \\
\hline 1 & $48(62.3)$ \\
\hline 2 & $10(13.0)$ \\
\hline 3 & $3(3.9)$ \\
\hline \multicolumn{2}{|l|}{ Tumor vol in $\mathrm{cm}^{3}$} \\
\hline Median & 5.6 \\
\hline Range & $0.3-17.5$ \\
\hline No. of patients $w /$ brain edema before SRS & $40(65.6)$ \\
\hline \multicolumn{2}{|l|}{ Median edema vol in $\mathrm{ml}$} \\
\hline Median & 14.6 \\
\hline Range & $0.3-130.8$ \\
\hline \multicolumn{2}{|l|}{ Tumor locations } \\
\hline Parasagittal & $43(55.8)$ \\
\hline Falx & $34(44.2)$ \\
\hline \multicolumn{2}{|l|}{ Clinical symptoms } \\
\hline Headache & $22(28.6)$ \\
\hline Hemiparesis & $19(24.7)$ \\
\hline Seizure & $19(24.7)$ \\
\hline Visual disturbance & $13(16.9)$ \\
\hline Paresthesias & $8(10.4)$ \\
\hline Adjacent to venous sinus & $22(28.6)$ \\
\hline No. of tumors w/ upfront SRS & $15(19.5)$ \\
\hline No. of tumors w/ prior resection & $62(80.5)$ \\
\hline Simpson Grade 1 & $0(0)$ \\
\hline Simpson Grade 2 & $5(6.4)$ \\
\hline Simpson Grade 3 & $24(31.2)$ \\
\hline Simpson Grade 4 & $30(40.0)$ \\
\hline Simpson Grade 5 & $3(3.9)$ \\
\hline No. of tumors w/ cerebral edema prior to SRS & $47(61)$ \\
\hline \multicolumn{2}{|l|}{ MRI FU in mos } \\
\hline Median & 28 \\
\hline Range & $6-158$ \\
\hline \multicolumn{2}{|l|}{ Clinical FU in mos } \\
\hline Median & 36 \\
\hline Range & $6-158$ \\
\hline
\end{tabular}

parameters are described in Table 2. The Leksell Gamma Knife model U was used from 1991 until 2001, the model C from 2001 to 2007, and the Perfexion from 2007 to present. At the time of radiosurgery, most patients received a single dose of intravenous dexamethasone unless there was a contraindication to receiving this (e.g., poorly controlled diabetes). The median prescription dose was 15 
TABLE 2. Stereotactic radiosurgery parameters

\begin{tabular}{lcc}
\hline \multicolumn{1}{c}{ SRS Treatment Parameters } & Mean & Range \\
\hline Margin radiation dose in Gy & 15 & $10-20$ \\
\hline Maximum radiation dose in Gy & 30 & $24-60$ \\
\hline Isodose level (\%) & 50 & $30-55$ \\
\hline
\end{tabular}

Gy (range 10-20 Gy), prescribed to a median 50\% isodose line (range 30\%-55\%) (Table 2).

\section{Clinical and Radiographic Follow-Up}

MRI and clinical follow-up were performed at approximately 6-month intervals for the first 2 years after GKRS with subsequent annual follow-up thereafter. All followup imaging was reviewed by both a neuroradiologist and a neurosurgeon at our institution. Because our institution is a tertiary referral center, clinical follow-up was performed through a combination of outpatient visits to our clinic and inpatient admissions to our hospital, outpatient visits from referring primary care physicians, and inpatient admissions to referring hospitals. For the 61 patients (77 tumors), the median duration of imaging follow-up was 28 months (range 6-158 months), and the median duration of clinical follow-up was 36 months (range 6-158 months).

\section{Tumor and Edema Volumetric Assessment}

The volumes of tumor and edema were determined for each imaging data set available for patients in a longitudinal fashion. Edema volume was determined from T2weighted MRI, and the tumor volume was determined from postcontrast T1-weighted imaging. Volumes were computed by segmenting the edema and tumors on a slice-by-slice basis and numerically integrating the resulting calculated areas using the trapezoidal rule. ${ }^{23}$ ImageJ software (National Institutes of Health) was used for contouring and volume computations. Tumor regression was defined as a reduction of the tumor volume by more than $10 \%$ of its volume at the time of GKRS. Tumor progression was defined as an increase in the tumor volume by more than $10 \%$ of the GKRS volume. A tumor was considered stable if the lesion was within $\pm 10 \%$ of its volume at the time of GKRS. The 10\% variation for defining an unequivocal change was selected in part based upon volumetry error estimation previously performed by our group over the range of lesion volumes commonly found in single-session GKRS. ${ }^{23}$

The edema index has been previously used to study edema response around benign and malignant tumors including meningiomas and metastases., ${ }^{7,1719}$ At each time point for which an MR image was available, the mean edema index for a tumor was computed as follows: mean edema index $=($ volume of peritumoral edema $) /($ volume of tumor).

For the purposes of this study, we defined a regression in edema as a consistent reduction in the edema index of more than $10 \%$ from its initial value at the time of GKRS. Stable edema was defined as an edema index of $\pm 10 \%$ of its value at the time of GKRS. Edema was classified as progressive followed by regressive for tumors that ex- hibited an edema index of greater than $10 \%$ of its GKRS value at any follow-up point, but eventually demonstrated a decrease in the edema index of greater than $10 \%$ of its value at the time of GKRS. Progression of edema was defined as a persistent increase in the edema index by more than $10 \%$ as seen on each of the post-GKRS MR images. Patients with no apparent T2-weighted changes surrounding the tumor were described as having no edema.

For all meningiomas included in this study, the edema index was computed until last follow-up MRI or until the time of resection if one was undertaken for tumor growth with or without edema.

\section{Statistical Analysis}

Descriptive statistics for all data were presented as the median and range for continuous variables and as frequency and percentages for categorical variables. Logistic regression modeling was used to analyze the prognostic factors of post-SRS brain edema and to assess odds ratios. Potential prognostic factors investigated included age, sex, tumor volume, tumor location, prior resection, venous sinus invasion, prior brain edema, margin dose, and maximum dose. Factors with a $\mathrm{p}$ value $<0.25$ in univariate analysis were eligible to enter into multivariate analysis by forward selection. ${ }^{1}$ Edema indexes of different follow-up points were plotted to demonstrate the quantitative changes of brain edema. Pre-SRS tumor volume and post-SRS maximal edema index were further assessed by linear regression to model the relationship between initial tumor size and consequential peritumoral edema. All statistical tests were 2 -sided, and $\mathrm{p}<0.05$ was considered statistically significant. All statistical analyses were performed using SPSS (version 20.0, IBM).

\section{Results}

\section{Tumor Control and Edema}

The median radiographic follow-up in this cohort of 77 meningiomas was 28 months (range 6-158 months). Using the Kaplan-Meier approach, progression-free survival at 2,3 , and 5 years was $98 \%, 90 \%$, and $90 \%$, respectively. The edema index progressed in $75 \%$ of enlarging tumors. In $25 \%$ of enlarging tumors, the edema index was stable despite an increase in tumor volume.

\section{New or Worsening Edema After Radiosurgery}

Following GKRS, edema developed or progressed in association with 31 tumors (40\%) (Table 3). New or worsening edema as denoted by the maximum edema index for all treated tumors peaked at 36 months after GKRS (Fig. 1).

Of the 31 tumors with new or progressive edema, 20 tumors had a progression of the peritumoral edema followed by eventual regression of the edema at last follow-up. For tumors that demonstrated initial edema progression followed by regression, the median interval between GKRS and peak edema was 18 months (range 6-24 months). For tumors with progressive edema at last follow-up, the median interval between GKRS and peak of the edema was longer at 36 months. Of those with progressive tumor and/ or edema after GKRS, 11 tumors (14\%) at last follow-up underwent a craniotomy and resection. 
TABLE 3. Imaging outcomes after SRS

\begin{tabular}{lc}
\hline Pattern of Edema Changes $^{*}$ & No. of Tumors (\%) \\
\hline Regression & $19(25)$ \\
\hline Stable & $18(23)$ \\
\hline Progression, then regression & $20(26)$ \\
\hline Progression & $11(14)$ \\
\hline No edema peri-SRS & $9(12)$ \\
\hline
\end{tabular}

* Regression: edema index decrease persistently $>10 \%$; Stable: edema index decrease or increase within $\pm 10 \%$; progression, then regression: edema index increase $>10 \%$ and then decrease $>10 \%$ compared to original edema index; progression: edema index increase persistently $>10 \%$; no edema: edema index $=0$ during the period of SRS and follow-up.

Regression of preexisting edema occurred in 19 tumors. The preexisting edema remained stable in 18 (23\%) of tumors at last follow-up. Edema was absent at the time of the GKRS and did not develop in 9 tumors (12\%).

\section{Factors Related to New or Worsening Edema}

We evaluated factors related to new or progressive edema after GKRS (Table 4). In univariate analysis, no prior resection was related to development of new or worsening edema ( $\mathrm{p}=0.041)$. Also, tumors with preexisting edema were more likely to develop persistent, progressive edema following radiosurgery $(\mathrm{p}=0.001)$. There was a trend toward significance in univariate analysis for tumors with a volume of more than $10 \mathrm{~cm}^{3}$ to develop new or worsening edema $(p=0.057)$.

In multivariate analysis, new or worsening edema was associated with an initial tumor volume greater than 10 $\mathrm{cm}^{3}(\mathrm{p}=0.033)$, absence of prior resection $(\mathrm{p}=0.026)$, prior existing peritumoral edema $(\mathrm{p}=0.001)$, higher radiosurgical margin dose $(\mathrm{p}=0.016)$, and higher maximum dose $(\mathrm{p}=0.048)$. A linear relationship was noted between initial tumor volume and maximal tumor edema index (Fig. 2; $\mathrm{p}=0.037, \mathrm{R}^{2}=0.09$ ). Similarly, there was a statistically significant relationship between initial edema index and maximal tumor edema index in the post-GKRS follow-up period (Fig. 3; $p<0.001, \mathrm{R}^{2}=0.46$ ).

\section{Corticosteroid Use in the Post-GKRS Setting}

In the post-GKRS period, 16 patients were placed on a temporary course of steroids for new or worsening edema. Given the parasagittal and parafalcine location of the meningiomas in this study, the symptoms in such patients most often included headaches but occasionally included seizures or weakness. Of those, 7 patients had radiographic and clinical improvement of their edema with a short course of steroids.

\section{Discussion}

Peritumoral edema occurs in $11 \%-92 \%$ of meningiomas at baseline., ${ }^{2,13,18}$ Patients with significant mass effect and edema associated with a meningioma frequently undergo resection, but this resection may be subtotal in nature. Those meningioma patients selected for SRS may in fact be less likely to exhibit peritumoral edema than those who undergo resection. However, radiosurgery is still used

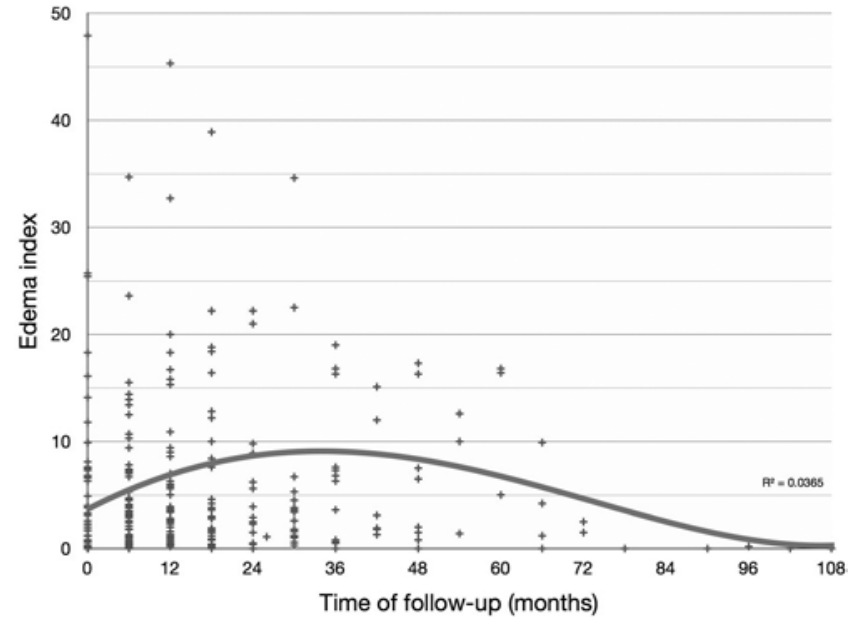

FIG. 1. Edema index for parasagittal or parafalcine meningiomas following GKRS. The individual values available at each time point are denoted by a plus sign. The curve represents a best fit of the edema indices with a peak at approximately 36 months following GKRS.

at times for treating patients who exhibit peritumoral edema, particularly if it is mild or if associated symptoms are controlled with corticosteroids.

After SRS, reports of new or worsening peritumoral edema have been reported in $14 \%-25 \%$ of patients. . $^{2,3,10,11}$ This edema usually occurs in a delayed fashion after radiosurgery. In a study by Kondziolka et al., the onset of edema typically occurred 6-8 months after SRS (range 1-23 months). ${ }^{12}$ However, in a study by Cai et al., the median time to onset of new or worsening edema was slightly earlier at 4 months, but the at-risk period was longer (range 1.5-48 months). ${ }^{2}$ Kan et al. found that the mean time to edema was 5.5 months after SRS and the mean duration was 16 months. ${ }^{10}$ Post-SRS edema has been associated with tumor volume, preexisting edema, tumor-brain contact area, and radiation dose. . $^{2,3,10,11}$ In the current study, we observed new or worsening edema in $40 \%$ of patients, of whom $26 \%$ eventually had edema regression but $14 \%$ had persistence of the edema after radiosurgery. We found that an elevated edema index frequently started 6 months post-GKRS, and the maximum edema index in our study occurred 36 months after GKRS (Fig. 1). The time interval for onset of edema and peak of the edema represent a combination of those patients with progressive and persistent edema as well as those who have initially progressive edema followed by eventual regression of the edema and usually tumor regression as well.

In the current study, patients with a tumor volume greater than $10 \mathrm{~cm}^{3}$, preexisting brain edema, no prior resection, and treatment with a higher dose were more likely to experience progressive edema following radiosurgery. Others have found that radiosurgery for meningiomas exceeding $10 \mathrm{~cm}^{3}$ have been associated with a worse prognosis after radiosurgery. ${ }^{4}$ The current study and prior ones suggest but do not irrefutably demonstrate that resection, even if incomplete, may be of benefit to such patients if it will enable a reduction in the tumor volume below 10 $\mathrm{cm}^{3}$ and eliminate the edema at the time of radiosurgery delivery. ${ }^{4}$ Practically, less than one-fourth of all cases of 
TABLE 4. Prognostic factors associated with post-SRS edema including the condition of stationary, progressive, and progressive-regressive brain edema after SRS

\begin{tabular}{lccccc}
\hline \multirow{2}{*}{ Variable } & \multicolumn{2}{c}{ Univariate } & & \multicolumn{2}{c}{ Multivariate } \\
\cline { 2 - 3 } \cline { 6 - 6 } & p Value & OR $(95 \% \mathrm{Cl})$ & & $\mathrm{p}$ Value & OR $(95 \% \mathrm{Cl})$ \\
\hline Age & 0.66 & $0.99(0.96-1.03)$ & & & \\
\hline Sex (male vs female) & 0.32 & $1.61(0.63-4.13)$ & & \\
\hline Tumor vol (>10 vs $\left.\leq 10 \mathrm{~cm}^{3}\right)$ & 0.057 & $3.68(0.96-14.08)$ & & 0.033 & $12.8(1.24-133.4)$ \\
\hline Tumor location (parasagittal vs falx) & 0.44 & $0.69(0.27-1.76)$ & & \\
\hline Prior resection (yes vs no) & 0.041 & $0.60(0.36-0.98)$ & & 0.026 & $0.33(0.125-0.88)$ \\
\hline Venous sinus invasion (no vs yes) & 0.23 & $0.87(0.29-2.58)$ & & 0.128 & $8.37(0.54-129.1)$ \\
\hline Brain edema prior to SRS (no vs yes) & 0.001 & $0.65(0.27-1.01)$ & & 0.001 & $0.713(0.19-1.45)$ \\
\hline Margin dose & 0.18 & $1.00(0.93-1.08)$ & & 0.016 & $1.786(1.11-2.86)$ \\
\hline Maximum dose & 0.22 & $0.99(0.92-1.08)$ & & 0.048 & $0.881(0.78-1.00)$ \\
\hline
\end{tabular}

involving a parasagittal/parafalcine meningioma lead to a Simpson Grade I removal, and this is particularly the case when the tumor invades the dura of the superior sagittal sinus. ${ }^{25}$ Although there is no definitive proof in the literature, it is interesting to consider the possibility that multisession radiosurgery for meningioma patients could afford a lower risk of new or worsening edema. In terms of radiation dose as a risk factor for edema, fractionation may offer some reduction in the risk of subsequent edema formation. ${ }^{6}$ In one study of patients with convexity and parasagittal meningiomas, fractionated SRS was associated with less risk of posttreatment edema than SRS. ${ }^{6}$ In another study by Unger and colleagues, the authors found that post-SRS edema for meningiomas was associated with single fraction as compared with multifraction SRS (2 or more fractions). ${ }^{26}$ In a study evaluating edema after SRS or radiotherapy, Kalapurakal et al. demonstrated a statistically significant increase in edema when radiation was delivered in more than 6 Gy per fraction. ${ }^{9}$ In a cohort of partially resected meningiomas treated with radiation therapy at the University of California, San Francisco, there were no cases of posttreatment edema. ${ }^{1}$ The potential benefits of fractionation in terms of reducing postradiosurgical edema along with the alteration in the biological effective dose as it relates to tumor control will require more rigorous study.

Previous SRS studies have demonstrated a tendency for increased peritumoral edema in parafalcine and parasagittal meningiomas compared with skull base ones. ${ }^{3}$ Such edema has been associated with postradiosurgical morbidity.$^{13}$ Peritumoral edema associated with meningiomas is likely vasogenic in nature, although some contend that a cytotoxic component contributes to the edema as well.,29 Meningiomas with edema have been shown to exhibit blood vessels with hyperpermeability, and, thus, the tumor itself may be the source of at least some of the edema fluid. ${ }^{24}$ Such a pathophysiology for the peritumoral edema would necessitate partial breakdown of the tumorbrain interface as is often seen with irregular or widened enhancement of the tumor margin on postcontrast MR images. Moreover, temporary swelling of a parasagittal or parafalcine meningioma can compress the adjacent venous structures and lead to additional cerebral edema. Ischemic changes in edematous brain parenchyma may lead to expression of vascular endothelial growth factor (VEGF). VEGF receptors have also been shown to exhibit a significantly positive relationship to edema volume..$^{18,29}$ Combining these factors, some patients with a parafalcine or parasagittal meningioma may experience a period of worsening cerebral edema and accompanying neurological dysfunction following SRS.

Corticosteroids are most commonly used to reduce the peritumoral edema associated with meningiomas. The use of prophylactic anticonvulsants may also be considered depending upon the degree and location of the edema. VEGF inhibitors such as bevacizumab have been shown to reduce baseline peritumoral edema as well as edema following radiosurgery and radiation therapy. ${ }^{14,15} \mathrm{We}$ have also reported on the use of bevacizumab for arteriovenous malformations and have used it rarely in the setting of other benign intracranial pathologies. ${ }^{28}$ While bevacizumab was part of the current study, there is substantial reason to believe that it would be of therapeutic value for meningioma patients with worsening edema and particularly those who are intolerant of, or whose edema is refrac-

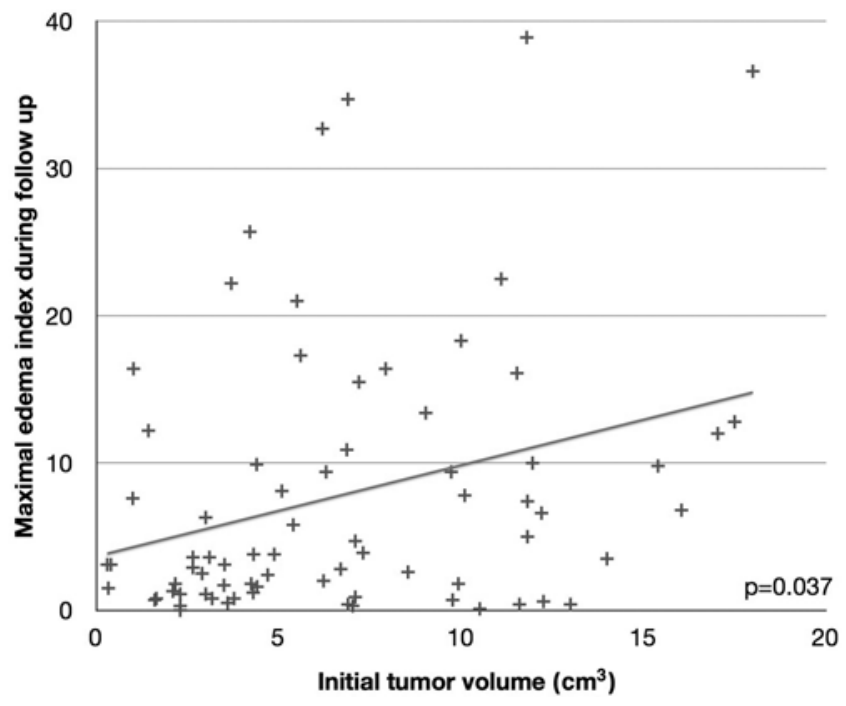

FIG. 2. Maximum edema index per patient is plotted against initial tumor volume at the time of GKRS. The line represents the best fit of tumor volume versus maximum edema index. 


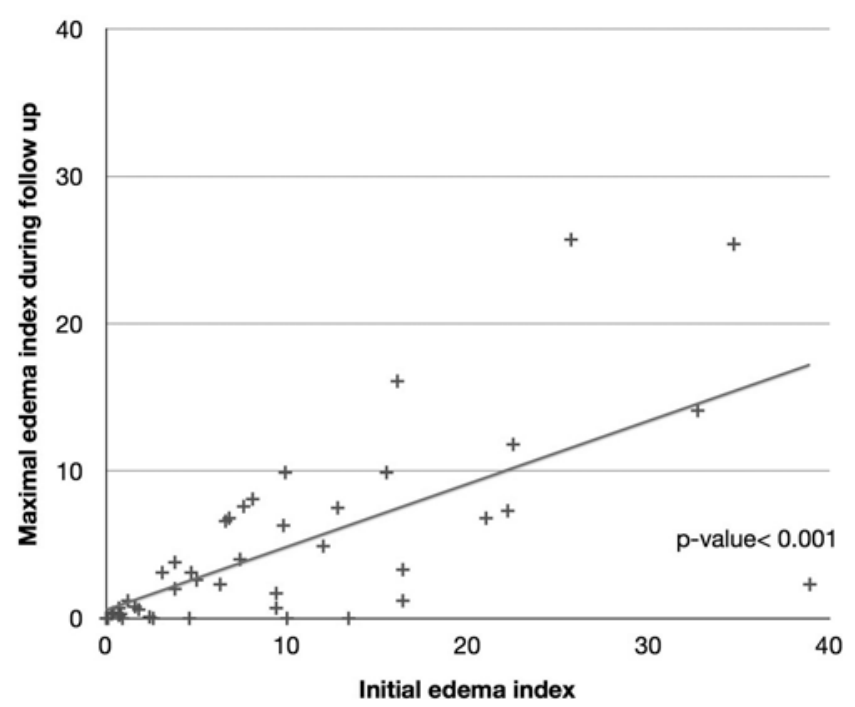

FIG. 3. The relationship between initial edema index and maximal edema index was statistically significant and a linear one.

tory to, corticosteroid treatment. Alternately, resection of a meningioma with worsening edema can be undertaken if necessary. However, it is rare for a meningioma treated with radiosurgery to necessitate resection for edema alone, as this edema is transient and is usually manageable with medical therapy.

\section{Study Limitations}

This study has limitations inherent to single-center, retrospective clinical trials. Patient selection, referral patterns from community centers to a tertiary care center, and evolving treatment algorithms have led to biases within the current study. Also, while $80.5 \%$ of patients had histological diagnosis as a WHO Grade I meningioma, the remaining patients were included on the basis of clinical and radiographic features. While a minority of the cohort, these patients without histological confirmation could have harbored tumor other than Grade I meningiomas, and, as such, introduce a study bias. In addition, the radiosurgical technique including the delivery device, dose planning software, neuroimaging protocols for stereotactic targeting, and dose selection have evolved over the study period.

We did not routinely perform MR venography or conventional angiography as part of pre- or post-GKRS assessment. Therefore, we could not evaluate this likely important factor of venous stasis or occlusion as a contributing factor to peritumoral edema. Volumetric segmentation of the tumor and edema are subject to well-known biases, particularly in the setting of smaller tumors where tomographic errors and contouring variations may lead to greater uncertainty. ${ }^{23}$ In addition, our volumetric criteria for tumor progression, regression, and stability do vary from other systems (e.g., RECIST). ${ }^{27}$ Also, as some patients were placed on a regimen of corticosteroids during this study, the presence of steroids could have confounded the measurements of the edema index. Further study of the phenomenon of postradiosurgical edema should be performed and is underway in a multicenter study.

\section{Conclusions}

Parasagittal and parafalcine meningiomas may develop new or worsening edema following SRS. In these 2 locations, temporary or permanent T2-weighted changes after radiosurgery are not rare. Progressive edema is related to the initial volume of the tumor. While the progressive edema occurs in a minority of patients, the occurrence of such edema nevertheless warrants longitudinal clinical and radiographic follow-up of treated patients. Intervention with medications or resection may be required for symptomatic and persistent edema following radiosurgery.

\section{Acknowledgments}

We thank Drs. Ahmed Awad and Francis Fezeu for assistance with this manuscript.

\section{References}

1. Barbaro NM, Gutin PH, Wilson CB, Sheline GE, Boldrey EB, Wara WM: Radiation therapy in the treatment of partially resected meningiomas. Neurosurgery 20:525-528, 1987

2. Cai R, Barnett GH, Novak E, Chao ST, Suh JH: Principal risk of peritumoral edema after stereotactic radiosurgery for intracranial meningioma is tumor-brain contact interface area. Neurosurgery 66:513-522, 2010

3. Chang JH, Chang JW, Choi JY, Park YG, Chung SS: Complications after gamma knife radiosurgery for benign meningiomas. J Neurol Neurosurg Psychiatry 74:226-230, 2003

4. DiBiase SJ, Kwok Y, Yovino S, Arena C, Naqvi S, Temple $\mathrm{R}$, et al: Factors predicting local tumor control after gamma knife stereotactic radiosurgery for benign intracranial meningiomas. Int J Radiat Oncol Biol Phys 60:1515-1519, 2004

5. Ding D, Xu Z, McNeill IT, Yen CP, Sheehan JP: Radiosurgery for parasagittal and parafalcine meningiomas. J Neurosurg 119:871-877, 2013

6. Girvigian MR, Chen JC, Rahimian J, Miller MJ, Tome M: Comparison of early complications for patients with convexity and parasagittal meningiomas treated with either stereotactic radiosurgery or fractionated stereotactic radiotherapy. Neurosurgery 62 (5 Suppl):A19-28, 2008

7. Gurkanlar D, Er U, Sanli M, Ozkan M, Sekerci Z: Peritumoral brain edema in intracranial meningiomas. J Clin Neurosci 12:750-753, 2005

8. Hasegawa T, Kida Y, Yoshimoto M, Iizuka H, Ishii D, Yoshida K: Gamma Knife surgery for convexity, parasagittal, and falcine meningiomas. J Neurosurg 114:1392-1398, 2011

9. Kalapurakal JA, Silverman CL, Akhtar N, Laske DW, Braitman LE, Boyko OB, et al: Intracranial meningiomas: factors that influence the development of cerebral edema after stereotactic radiosurgery and radiation therapy. Radiology 204:461-465, 1997

10. Kan P, Liu JK, Wendland MM, Shrieve D, Jensen RL: Peritumoral edema after stereotactic radiosurgery for intracranial meningiomas and molecular factors that predict its development. J Neurooncol 83:33-38, 2007

11. Kobayashi T, Kida Y, Mori Y: Long-term results of stereotactic gamma radiosurgery of meningiomas. Surg Neurol $\mathbf{5 5 : 3 2 5 - 3 3 1 , 2 0 0 1}$

12. Kondziolka D, Mathieu D, Lunsford LD, Martin JJ, Madhok $\mathrm{R}$, Niranjan A, et al: Radiosurgery as definitive management of intracranial meningiomas. Neurosurgery 62:53-60, 2008

13. Lee KJ, Joo WI, Rha HK, Park HK, Chough JK, Hong YK, et al: Peritumoral brain edema in meningiomas: correlations between magnetic resonance imaging, angiography, and pathology. Surg Neurol 69:350-355, 2008

14. Levin VA, Bidaut L, Hou P, Kumar AJ, Wefel JS, Bekele BN, 
et al: Randomized double-blind placebo-controlled trial of bevacizumab therapy for radiation necrosis of the central nervous system. Int J Radiat Oncol Biol Phys 79:1487-1495, 2011 (Erratum in Int J Radiat Oncol Biol Phys 84:6)

15. Lou E, Sumrall AL, Turner S, Peters KB, Desjardins A, Vredenburgh JJ, et al: Bevacizumab therapy for adults with recurrent/progressive meningioma: a retrospective series. J Neurooncol 109:63-70, 2012

16. Novotný J Jr, Kollová A, Liscák R: Prediction of intracranial edema after radiosurgery of meningiomas. J Neurosurg 105 Suppl:120-126, 2006

17. Osawa T, Tosaka M, Nagaishi M, Yoshimoto Y: Factors affecting peritumoral brain edema in meningioma: special histological subtypes with prominently extensive edema. J Neurooncol 111:49-57, 2013

18. Otsuka S, Tamiya T, Ono Y, Michiue H, Kurozumi K, Daido $\mathrm{S}$, et al: The relationship between peritumoral brain edema and the expression of vascular endothelial growth factor and its receptors in intracranial meningiomas. J Neurooncol 70:349-357, 2004

19. Pan HC, Sun MH, Chen CC, Chen CJ, Lee CH, Sheehan J: Neuroimaging and quality-of-life outcomes in patients with brain metastasis and peritumoral edema who undergo Gamma Knife surgery. J Neurosurg 109 Suppl:90-98, 2008

20. Patil CG, Hoang S, Borchers DJ III, Sakamoto G, Soltys SG, Gibbs IC, et al: Predictors of peritumoral edema after stereotactic radiosurgery of supratentorial meningiomas. Neurosurgery 63:435-442, 2008

21. Simis A, Pires de Aguiar PH, Leite CC, Santana PA Jr, Rosemberg S, Teixeira MJ: Peritumoral brain edema in benign meningiomas: correlation with clinical, radiologic, and surgical factors and possible role on recurrence. Surg Neurol 70:471-477, 2008

22. Singh VP, Kansai S, Vaishya S, Julka PK, Mehta VS: Early complications following gamma knife radiosurgery for intracranial meningiomas. J Neurosurg 93 (Suppl 3):57-61, 2000

23. Snell JW, Sheehan J, Stroila M, Steiner L: Assessment of imaging studies used with radiosurgery: a volumetric algorithm and an estimation of its error. Technical note. J Neurosurg 104:157-162, 2006
24. Stevens JM, Ruiz JS, Kendall BE: Observations on peritumoral oedema in meningioma. Part II: Mechanisms of oedema production. Neuroradiology 25:125-131, 1983

25. Sughrue ME, Kane AJ, Shangari G, Rutkowski MJ, McDermott MW, Berger MS, et al: The relevance of Simpson Grade I and II resection in modern neurosurgical treatment of World Health Organization Grade I meningiomas. J Neurosurg 113:1029-1035, 2010

26. Unger KR, Lominska CE, Chanyasulkit J, Randolph-Jackson P, White RL, Aulisi E, et al: Risk factors for posttreatment edema in patients treated with stereotactic radiosurgery for meningiomas. Neurosurgery 70:639-645, 2012

27. Warren KE, Patronas N, Aikin AA, Albert PS, Balis FM: Comparison of one-, two-, and three-dimensional measurements of childhood brain tumors. J Natl Cancer Inst 93:1401-1405, 2001

28. Williams BJ, Park DM, Sheehan JP: Bevacizumab used for the treatment of severe, refractory perilesional edema due to an arteriovenous malformation treated with stereotactic radiosurgery. J Neurosurg 116:972-977, 2012

29. Yoshioka H, Hama S, Taniguchi E, Sugiyama K, Arita K, Kurisu K: Peritumoral brain edema associated with meningioma: influence of vascular endothelial growth factor expression and vascular blood supply. Cancer 85:936-944, 1999

\section{Author Contributions}

Conception and design: Sheehan. Acquisition of data: Lee, $\mathrm{Xu}$, Przybylowski, Melmer. Analysis and interpretation of data: Sheehan, Lee, Xu. Drafting the article: Sheehan. Critically revising the article: Sheehan, Schlesinger. Reviewed submitted version of manuscript: Sheehan, Schlesinger. Approved the final version of the manuscript on behalf of all authors: Sheehan. Statistical analysis: Lee, Xu, Schlesinger. Administrative/technical/material support: Sheehan. Study supervision: Sheehan, Schlesinger.

\section{Correspondence}

Jason P. Sheehan, Department of Neurological Surgery, University of Virginia Health System, Box 800212, Charlottesville, VA 22908. email: jsheehan@virginia.edu. 